\title{
FATORES MOTIVACIONAIS ASSOCIADOS À PRÁTICA DE EXERCÍCIO FÍSICO EM ESTUDANTES UNIVERSITÁRIOS
}

\author{
MS. ROSIMEIDE FRANCISCO SANTOS LEGNANI \\ Mestre em Educação Física - Universidade Estadual de Londrina - UEL \\ Centro de Pesquisa em Exercício e Esporte da Universidade Federal do Paraná - UFPR \\ (Curitiba - Paraná - Brasil) \\ e-mail: legnanirosi@hotmail.com
}

\section{DR. DARTAGNAN PINTO GUEDES}

Doutor em Educação Física - Universidade Estadual de Londrina - UEL

(Londrina - Paraná - Brasil)

e-mail:darta@sercomtel.com.br

\section{MS. ELTO LEGNANI}

Doutorando pelo Departamento de Educação Física - Universidade Federal do Paraná Centro de Pesquisa em Exercício e Esporte da Universidade Federal do Paraná - UFPR

(Curitiba - Paraná - Brasil)

e-mail: legnanielto@hotmail.com

\section{GRAD. VALTER CORDEIRO BARBOSA FILHO}

Mestrando pelo Departamento de Educação Física - Universidade Federal do Paraná Centro de Pesquisa em Exercício e Esporte da Universidade Federal do Paraná - UFPR (Curitiba - Paraná - Brasil)

e-mail: valtercbf@gmail.com

\section{DR. WAGNER DE CAMPOS}

Professor do Departamento de Educação Física da Universidade Federal do Paraná Centro de Pesquisa em Exercício e Esporte da Universidade Federal do Paraná - UFPR

(Curitiba - Paraná - Brasil)

e-mail:wagner@ufpr.br

\begin{abstract}
RESUMO
O estudo identificou fatores motivacionais associados à prática de exercício físico em estudantes universitários mediante versão traduzida e adaptada do Exercise Motivation Inventory. Metodologia: participaram do estudo 63 sujeitos (20 e 30 anos). Resultados: Os fatores motivacionais mais importantes foram Prevenção de Doenças, Prazer/Bem-Estar e Condição Física. Diferenças significativas entre os gêneros foram observadas em cinco fatores
\end{abstract}


motivacionais: Afiliação $(p \leq 0,01)$, Competição $(p \leq 0,01)$, Controle do Peso Corporal ( $p \leq$ $0,04)$, Reabilitação da saúde ( $p \leq 0,03)$ e Reconhecimento Social $(p \leq 0,05)$. Conclusão: importantes aspectos motivacionais devem ser considerados em intervenções direcionadas à promoção da prática de exercício físico em estudantes universitários.

PALAVRAS-CHAVE: Motivação; atividade física; promoção de saúde; adulto jovem.

\section{INTRODUÇÃO}

A literatura sugere que a atividade física constitui fator determinante para o estado de saúde, independentemente de gênero, idade e nível socioeconômico (VUORI, 2004; BLAIR; CHENG; HOLDER, 200I). Estudos prévios apontam que a prática habitual de atividade física possa estar inversamente relacionada ao aparecimento e ao desenvolvimento de disfunções de caráter crônico-degenerativo, como é o caso da hipertensão, do diabetes e da aterosclerose (HASKELL; BLAIR; HILL, 2009; VUORI, 2004; BAUMAN, 2004).

Neste particular, o exercício físico representa um componente específico da atividade física direcionado ao aprimoramento e monitoração dos componentes equivalentes ao tipo, à frequência, à intensidade e à duração do esforço físico (CASPERSEN; POWELL; CHRISTENSON, 1985), isto tem sido considerado fundamental para conservação e promoção da saúde individual; sobretudo, em idades jovens.

$\bigcirc$ envolvimento com a prática de exercício físico pode resultar em importantes benefícios psicológicos e fisiológicos, como minimização de estresse, controle de peso corporal, aprimoramento das capacidades cardiorrespiratórias e musculoesqueléticas, o que repercute na prevenção de disfunções cardiovasculares e metabólicas (THOMPSON, 2009). De fato, Legnani (2009), destaca que os principais fatores motivacionais à prática do exercício físico em universitários são: a prevenção de doenças, o controle de peso corporal e a aparência física que são definidos como fatores extrínsecos, além disso, o controle do estresse, o prazer e o bem-estar que são definidos como fatores intrínsecos.

Embora seja consistente a importância do exercício físico para a saúde individual, diversos estudos têm constatado que a proporção de pessoas que optam pela prática de exercício físico é bastante reduzida (SALLIS et al., 2009; SISSON; KATZMARZYK, 2008). A menor participação das pessoas em programas de exercício físico pode estar alicerçada no campo motivacional, considerando que, para assumir atitudes com essa característica do comportamento humano por período de tempo suficiente longo, independente da finalidade a que se propõe, é crucial que haja envolvimento de atributos associados à motivação (DECl; RYAN, I985; ROSE; MARKLAND; PARFITT, 200 I; MALTBY; DAY, 200I). 
Na literatura nacional, alguns estudos realizados se concentraram em indicadores relacionados à aderência e às barreiras para a prática habitual de atividade física, bem como os benefícios psicológicos advindos da prática do exercício físico (EIRAS et al., 20 I 0; ROJAS, 2003; REICHERT, 2004; DESCHAMPS; DOMINGUES FILHO, 2005). No entanto, poucos estudos têm focado a identificação dos fatores motivacionais que possam estar relacionados à prática de exercício físico em adolescentes e adultos (ALVES et al., 2007; CASTRO et al., 2009; CASTRO et al., 20 I0).

Nesta perspectiva, o presente estudo teve como objetivo identificar os principais fatores motivacionais para a prática de exercício físico em estudantes universitários, de acordo com o sexo, pertencentes a uma instituição de ensino superior da região sul do Brasil.

\section{MATERIAIS E MÉTODOS}

Foram selecionados de maneira não-probabilística para participar do estudo 63 estudantes universitários (54\% de rapazes e 46\% moças), com idades entre 20 e 30 anos, matriculados no ano letivo 2007, nos cursos de graduação em psicologia $(n=17)$ e engenharia civil $(n=46)$ da Universidade Estadual de Londrina, Paraná, Brasil. A participação dos estudantes universitários no estudo foi autorizada mediante assinatura do Termo de Consentimento Livre e Esclarecido. A coleta de dados foi realizada durante o mês de novembro de 2007, após aprovação de seu delineamento pelo Comitê de Ética em Pesquisa da Universidade (Parecer n. 2। 4/07 - CAAE - 02 15.0.268.000-07).

Para identificação dos fatores motivacionais associados à prática de exercício físico foi utilizado o instrumento Exercise Motivations Inventory-2 (EMI-2). Este instrumento contempla uma variedade de fatores de motivação (intrínsecos e extrínsecos) para a prática de exercício físico e foi originalmente idealizado em língua inglesa (MARKLAND; HARDY, I993; MARKLAND; INGLEDEW, 1997). Para o estudo foi utilizada a versão traduzida e adaptada transculturalmente para a realidade brasileira (LEGNANI, 2009), constituída por 44 questões distribuídas em 10 fatores motivacionais: Prazer e Bem-Estar; Controle do Estresse; Reconhecimento Social; Afiliação; Competição; Reabilitação da saúde; Prevenção de Doenças; Controle do Peso Corporal; Aparência Física; e Condição Física. Cada questão do instrumento é apresentada mediante escala de pontuação Likert com valores entre 0 a 5 , em que o valor mínino representa "nada verdadeiro" e o valor máximo "muito verdadeiro".

A partir disso, foi possível identificar o grau de importância dos motivos individuais para a prática de exercício físico. Para tanto, assumiu-se ponto de corte arbitrário equivalente a " 2,5 " (ponto intermediário do continuum de valores entre 
0 e 5) na escala de medida de respostas do EMI-2, o que corresponde a uma referência equidistante entre os escores extremos na escala. Desta forma, os fatores com valores médios superiores a "2,5" foram considerados os principais fatores de motivação para a prática de exercício físico entre os estudantes universitários.

Recursos da estatística descritiva foram utilizados para análise dos dados. A normalidade dos dados foi testada por meio do teste de Kolmogorov-Smirnov (KS). Nas variáveis que apresentaram distribuição normal, foi utilizado o teste $t$ de Student para amostras independentes, para identificar diferenças significativas entre os sexos, com relação a cada fator de motivação para a prática de exercício físico. Nas demais variáveis, foi aplicado um teste $U$ de Mann Whitney. Os dados foram analisados por meio do pacote computadorizado Statistical Package for the Social Sciences (SPSS), versão 15.0.

\section{RESULTADOS}

A ordem hierárquica dos escores médios obtidos para os fatores motivacionais associados à prática de exercício físico dos estudantes universitários é apresentada na Figura I. Quatro fatores motivacionais apresentaram escores médios superiores ao ponto-de-corte "2,5": Prevenção de Doenças (3,52 $\pm 1,1$ ), Prazer e Bem-Estar (3,37 $\pm 1,3)$, Condição Física $(3,06 \pm 1,0)$ e Controle do Estresse $(2,93$ \pm I,3 I). Os fatores motivacionais com menores escores médios foram Reconhecimento Social $(0,94 \pm 1,1)$, Reabilitação da saúde (1,42 \pm I ,4), Competição ( I,64 \pm 1,67) e Aparência Física $(1,63 \pm 0,9)$.

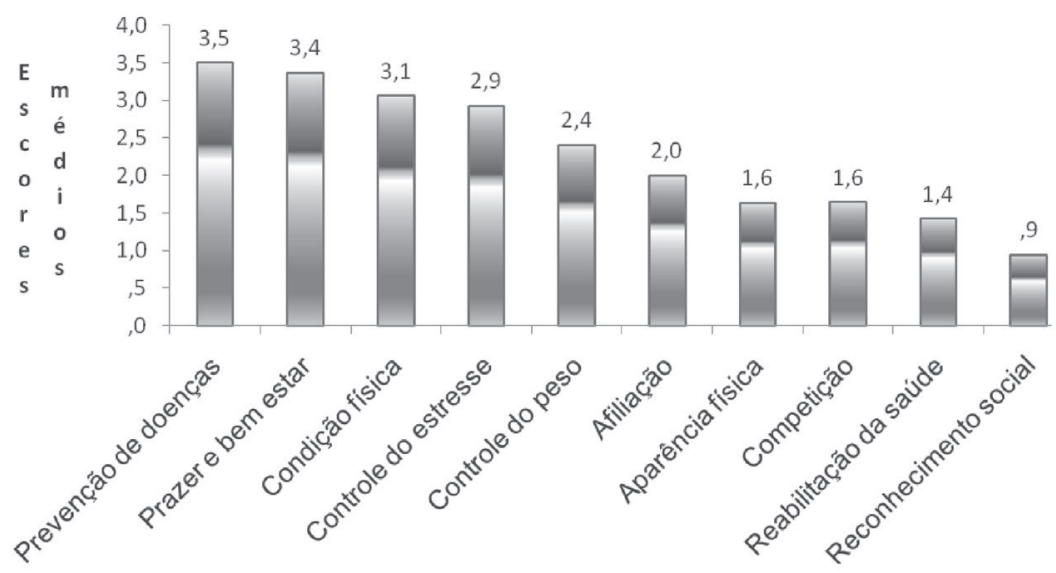

Figura I: Ordem hierárquica dos fatores motivacionais associados à prática de exercício físico em estudantes universitários. 
Na Tabela I são apresentados valores médios e desvios-padrão dos fatores motivacionais associados à prática de exercício físico separadamente por gênero. Dos 10 fatores motivacionais considerados no estudo, quatro deles (Prazer e Bem-Estar, Controle do Estresse, Prevenção de Doenças e Condição Física) apresentaram em ambos os gêneros valores médios superiores ao ponto de corte "2,5"; portanto, podem ser considerados igualmente nas moças e nos rapazes importantes fatores de motivação para a prática de exercício físico. Nas moças, por sua vez, o Controle do Peso Corporal também foi considerado importante fator motivacional associado à prática de exercício físico, isso não foi observado entre os rapazes.

Diferenças estatisticamente significativa entre os gêneros foram observadas em cinco fatores motivacionais: Afiliação ( $p \leq 0,0 \mathrm{I})$, Competição $(p \leq 0,0 \mathrm{I})$, Controle do Peso Corporal ( $p \leq 0,04)$, Reabilitação da saúde $(p \leq 0,03)$ e Reconhecimento Social ( $p$ $\leq 0,05)$. Neste particular, entre os rapazes, nota-se que o escore mais expressivo, foi observado no fator motivacional Competição $(2,38 \pm 1,78)$. Entre as mulheres, no fator motivacional Controle do Peso Corporal $(2,97 \pm 1,79)$. Isso demonstra que, dois fatores motivacionais de caráter extrínseco e de natureza distinta, parecem discriminar os gêneros quanto aos motivos que podem estar relacionados à prática de exercícios físicos.

Tabela I: Escores médios e desvios-padrão dos fatores de motivação para a prática de exercício físico de acordo com gênero dos estudantes universitários.

\begin{tabular}{|c|c|c|c|c|}
\hline \multirow[b]{2}{*}{ Fator de Motivação } & \multicolumn{2}{|c|}{ Média \pm Desvio padrão } & \multirow{2}{*}{$\begin{array}{l}\text { Estatística } \\
\text { (t ou U*) }\end{array}$} & \multirow[b]{2}{*}{ p } \\
\hline & $\begin{array}{l}\text { Rapazes } \\
(n=34)\end{array}$ & $\begin{array}{l}\text { Moças } \\
(n=29)\end{array}$ & & \\
\hline Prazer e bem estar & $3,56 \pm 1,33$ & $3,07 \pm|, 3|$ & $t=1,25$ & ns \\
\hline Controle do estresse & $2,75 \pm 1,34$ & $3,10 \pm 1,27$ & $t=1,14$ & ns \\
\hline Afiliação & $2,29 \pm 1,15$ & $1,62 \pm 0,84$ & $t=2,48$ & $p \leq 0,0$ I \\
\hline Aparência física & $1,65 \pm 1,03$ & $1,70 \pm 0,83$ & $t=1,45$ & ns \\
\hline Competição & $2,38 \pm 1,78$ & $0,78 \pm 1,03$ & $U=237,0$ & $p \leq 0,0$ I \\
\hline Condição física & $3,29 \pm 0,97$ & $2,88 \pm 1,05$ & $U=369,0$ & ns \\
\hline Controle do peso corporal & $2,00 \pm 1,86$ & $2,97 \pm 1,79$ & $U=345,0$ & $p \leq 0,04$ \\
\hline Prevenção de doenças & $3,41 \pm 1,23$ & $3,66 \pm 1,05$ & $U=449,0$ & ns \\
\hline Reabilitação da saúde & $1,05 \pm 1,24$ & $1,95 \pm 1,58$ & $U=340,5$ & $p \leq 0,03$ \\
\hline Reconhecimento social & $1,26 \pm 1,39$ & $0,63 \pm 0,77$ & $U=395,5$ & $p \leq 0,05$ \\
\hline
\end{tabular}

* $\mathrm{t}=$ teste $\mathrm{t}$ para amostras independentes (dados com distribuição normal); $\mathrm{U}=$ teste $\mathrm{U}$ de Mann-Whitney (dados com distribuição não-normal)

\section{DISCUSSÃO}

A inserção do jovem na universidade é considerada período crítico quanto à sua participação em programas de exercício físico e na prática habitual de atividade 
física (FONTES; VIANNA, 2009). A maioria dos jovens tende a abandonar a prática de exercício físico durante o período de permanência na universidade, devido à necessidade de se dedicar mais tempo ao estudo e ao trabalho, consequentemente, reduzindo a disponibilidade de tempo livre e de lazer (VELÁSQUEZ; TORRESNEIRA; SÁNCHEZ-MARTÍNEZ, 2006).

A identificação dos motivos que levam os indivíduos a praticar exercício físico pode ser importante no sentido de nortear ações de promoção de um estilo de vida saudável e ativo fisicamente no âmbito universitário (DE MOOR et al., 2006; MOUTÃO et al., 2003; BIDDLE; MUTRIE, 2007; CLARO; MONTEIRO, 2008). Embora a prática do exercício físico seja mediada por fatores ambientais, sociais e psicológicos, acredita-se que os fatores psicológicos sejam preponderantes para manutenção deste comportamento. Neste contexto, a motivação parece ser um dos principais componentes que levam as pessoas a se exercitarem regularmente.

Desse modo, a teoria da Autodeterminação procura caracterizar a motivação em duas dimensões: motivação extrínseca e intrínseca. A motivação extrínseca é caracterizada pela sua estreita identificação com reconhecimento social, premiações e recompensas. Por outro lado, a motivação intrínseca é considerada a forma mais autônoma de motivação e refere-se à tendência inata do ser humano na busca de novidades e desafios, como a capacidade de aprender e explorar a si próprio em relação ao exercício físico, sem preocupação em receber recompensas ou gratificações externas (RYAN; DECl, 2000).

Diante destes conceitos, dentre os quatro fatores motivacionais destacados pelos estudantes universitários como os mais importantes para a prática de exercício físico, encontram-se dois fatores extrínsecos (Prevenção de Doenças, Condição Física) e dois fatores de ordem intrínseca (Prazer e Bem-Estar e Controle do Estresse). Estes achados são consistentes com estudos prévios realizados em adultos jovens, universitários ou não-universitários, que identificaram os cuidados preventivos com a saúde, o prazer, o bem-estar e o controle do estresse como as dimensões que mais os motivavam para a prática de exercício físico (CASTRO et al., 20 I 0; KILPATRICK; HEBERT; BARTHOLONEW, 2005; NETZ; RAVIV, 2004; MARTINEZ-LÓPEZ, 2003; CASH; NOVY; GRANT, 2004).

Comparando ambos os gêneros foi identificada disposição discretamente diferente na ordem hierárquica dos fatores intrínsecos e extrínsecos para a prática de exercício físico. Entre as moças, os escores médios mais elevados foram observados nos fatores Prevenção de Doenças (extrínseco), Controle do Estresse (intrínseco), Prazer e Bem-Estar (intrínseco) e Controle do Peso Corporal (extrínseco), enquanto entre os rapazes destacaram os fatores Prazer e Bem-Estar (intrínseco), Prevenção de Doenças (extrínseco), Condição Física (extrínseco) e Controle do Estresse (intrínseco). 
Esses resultados corroboram com outros achados disponibilizados na literatura (BUCKWORTH et al., 2007).

De acordo com Hirschbruch, Fisberg e Mochizuki (2008), a maioria das pessoas, especialmente as moças, exercita-se para emagrecer ou controlar o peso corporal, sobretudo, pela supervalorização do corpo magro na sociedade atual. Essa afirmação pode ser confirmada pelo elevado escore médio apresentado pelas moças selecionadas no estudo equivalente ao fator motivacional Controle do Peso Corporal. Esses achados são divergentes dos encontrados por Castro et al., (20 I 0), que destacaram como fatores mais importantes para permanência em programas de exercícios os fatores relacionados ao "Bem-Estar Pessoal, Prevenção de Doenças e o Condicionamento Físico". A importância atribuída pelas mulheres foi superior a dos homens nos três fatores citados.

Os resultados do presente estudo indicam que, para promoção da prática de exercício físico no âmbito universitário, devem ser utilizados recursos capazes de mobilizar tanto aspectos relacionados à motivação intrínseca quanto à motivação extrínseca, principalmente o fator motivacional relacionado à Prevenção de Doenças e Condição Física, por terem apresentado escores médios elevados entre os estudantes universitários de ambos os sexos.

Esse fato pode ser justificado em razão da disseminação, na população, da importância do exercício físico para a saúde (KNUTH et al., 2009). Além disso, as eventuais agressões orgânicas induzidas pelo sedentarismo, como o aparecimento e o desenvolvimento de doenças crônico-degenerativas e osteomusculares, são percebidas, principalmente, após início da fase adulta, o que pode contribuir para modificação dos comportamentos e das atitudes diante da prática de exercício físico (NETZ; RAVIV, 2004; XU; BIDDLE, 2000; GILL et al., 1996). Esta hipótese é ratificada nos estudos prévios de Mazo; Cardoso; Aguiar, (2006) e Freitas et al., (2007), que identificaram questões relacionadas a melhora da saúde física, mental e a prevenção de doenças, como os principais motivos para a prática de exercício físico em idosos.

Finalmente, acredita-se que o estudo pode contribuir para identificar e entender os principais fatores que podem motivar a população universitária a praticar exercício físico, uma vez que, a maioria dos estudos disponibilizados na literatura investigou indicadores de aderência e as barreiras para a prática habitual de atividade física. Considerando o tamanho amostral e a utilização de uma amostra nãoprobabilística, sugere-se cautela na interpretação e generalização dos resultados.

\section{CONSINDERAÇÕES FINAIS}

De um modo geral, os fatores motivacionais relacionados à prática do exercício físico, que apresentaram ao escores médios mais elevados foram: Prevenção de Doenças, Prazer e bem estar e Condição Física. 
Entre as moças o fator motivacional extrínseco, Prevenção de Doenças, e entre os rapazes, o fator motivacional intrínseco, Prazer e Bem-Estar, foram considerados os fatores motivacionais mais relevantes para a prática de exercício físico no grupo investigado. Dessa forma, diferenças significativas entre os gêneros foram encontradas nos escores médios equivalentes aos fatores motivacionais, Afiliação, Competição, Controle do peso corporal, Reabilitação da Saúde e Reconhecimento Social.

Os resultados apontam para importantes aspectos motivacionais a serem considerados em ações intervencionistas voltadas à promoção da prática de exercício físico neste grupo específico de indivíduos. Logo, o presente estudo é uma tentativa de entender os principais fatores motivacionais relacionados à prática de exercício físico no âmbito universitário, de acordo com as dimensões que compõe o instrumento EMI-2 em sua versão adaptada para a realidade brasileira.

Portanto, ações que tenham como objetivo de promover a prática de exercícios físicos em universitários deve considerar os principais aspectos motivacionais e as diferenças entre os sexos, identificadas nesse estudo, como fatores importantes que podem potencializar o grau de motivação desses indivíduos quanto à sua participação em programas de exercícios físicos.

\section{Motivational factors associated with physical exercise in college students}

ABSTRACT: The study identified motivational factors associated with physical exercise in college students through translated and adapted version of the Exercise Motivations Inventory. Methodology: the participants were 63 students (20 and 30 years). Results: The main motivating factors for physical exercise were Prevention of Disease, Pleasure/Nell-being and Physical Condition. Significant differences between genders were observed in five motivational factors: Affiliation ( $p \leq$ 0.0 I), Competition ( $p \leq 0.01$ ), Body Weight Control ( $p \leq 0.04)$, Rehabilitation of health $(p \leq 0.03)$ and Social Recognition ( $p \leq 0.05)$. Conclusion: important motivational aspects to be considered in interventions aimed at the promotion of regular physical exercise in this group of individuals. KEYWORDS: Motivation; physical activity; health promotion; young adult.

\section{Factores de motivación para el ejercicio físico en estudiantes universitarios}

RESUMEN: El estudio identificó los factores motivacionales asociados con ejercicio fisico en estudiantes universitarios a través de la versión traducida y adaptada del Exercise Motivations Inventory. Metodología: participaron del estudio 63 estudiantes (20 y 30 años). Resultados: Los principales factores de motivación fueron Prevención de la Enfermedad, Placer/Bienestary Condición Física. Diferencias entre sexos se observó en cinco factores de motivación: Afiliación $(p \leq 0,0 I)$, Competencia $(p \leq 0,0 I)$, Control de peso corporal $(p \leq 0,04)$, Rehabilitación de la salud $(p \leq 0,03)$ y el Reconocimiento social $(p \leq 0,05)$. Conclusiones: importantes aspectos motivacionales debe ser considerada en las intervenciones dirigidas a la promoción del ejercicio físico en este grupo de individuos.

PALABRAS CLAVES: Motivación; actividad fisica; promoción de la salud; adulto joven. 


\section{REFERÊNCIAS}

ALVES, M. P. et al. Motivos que justificam a adesão de adolescentes à prática da natação: qual o espaço ocupado pela saúde? Revista Brasileira de Medicina do Esporte, Niterói, v. 13 , n. 6, p. 421-26, nov./dez. 2007.

BAUMAN, A. E. Updating the evidence that physical activity is good for health: an epidemiological review 2000-2003. Journal of Science and Medicine in Sport/Sports Medicine Australia, Austrália, v. 7, supl. I, p. 6-19, apr. 2004.

BIDDLE, S.; MUTRIE, N. Psychology of physical activity: Determinants, well-being and interventions. London: Routledge. 2007.

BLAIR, S. N.; CHENG, Y.; HOLDER, S. Is physical activity or physical fitness more important in defining health benefits? Medicine and Science in Sports and Exercise, Indianapolis, v. 33, n. 6, p. 379-399, Jun. 2001.

BUCKWORTH, J. et al. Decomposing intrinsic and extrinsic motivation for exercise: Application to stages of motivational readiness. Psychology of Sport and Exercise, Amsterdam, v. 8, n. 4, p. 44 I-46I, jul. 2007.

CASH, T. F.; NOVY, P. L.; GRANT, J. R. Why do women exercise? Factor analisys and further validation of the reasons for the exercise inventory. Perceptual and Motor Skills, Missoula, v. 78, n. 2, p. 539-544, apr. 2004.

CASPERSEN, C. J.; POWELL, K. E.; CHRISTENSON, G. M. Physical activity, exercise, and physical fitness: definitions and distinctions for health-related research. Public Health Reports, Boston, v. 100, n. 2, p. 126-131, mar./apr. 1985.

CASTRO, M. S. et al. Motivos de Ingresso nos Programas de Exercício Físicos Oferecidos pelo Serviço Social do Comércio - SESC-DF, Revista Movimento, Porto Alegre, v. I 5, n. 02 , p. 87-102, abr./jun. 2009.

CASTRO, M. S. et al. Motivos de permanência dos praticantes nos programas de exercícios físicos oferecidos pelo Serviço Social do Comércio - Brasil, Revista Motricidade, Santa Maria da Feira, v. 6, n. 4, p. 23-33, out./dez. 2010.

CLARO, R. M.; MONTEIRO, C. A. Vigilância de fatores de risco para doenças crônicas por inquérito telefônico nas capitais dos 26 estados brasileiros e no Distrito Federal (2006). Revista Brasileira de Epidemiologia, São Paulo, v. II , supl. I , p. 20-37, maio 2008.

DE MOOR, M. H. et al. Regular exercise, anxiety, depression and personality: a populationbased study. Preventive Medicine, New York, v. 42, n. 4, p. 273-279, apr. 2006.

DECI, E. L.; RYAN, R. M. Intrinsic Motivacion and Self-Determination in Human Behavior. New York: Plenum. 1985. 
DESCHAMPS, S. R.; DOMINGUES FILHO, L. A. Motivos e benefícios psicológicos que levam os indivíduos dos sexos masculino e feminino a praticarem o ciclismo indoor. Revista Brasileira Ciência e Movimento, Taguatinga, v. 13, n. 2, p. 27-32, ago. 2005.

EIRAS, S. B. et al. Fatores de adesão e manutenção da prática de atividade física por parte de idosos. Revista Brasileira de Ciências do Esporte, Campinas, v. 3I, n. 2, p. 75-89, jan. 2010.

FONTES, A. C. D.; VIANNA, R. P. T. Prevalência e fatores associados ao baixo nível de atividade física entre estudantes universitários de uma universidade pública da região Nordeste - Brasil. Revista Brasileira de Epidemiologia, São Paulo, v. 12, n. I, p. 20-29, mar. 2009.

FREITAS, C. M. S. M. et al. Aspectos motivacionais que influenciam a adesão e manutenção de idosos a programas de exercícios físicos. Revista Brasileira de Cineantropometria e Desempenho Humano, Florianópolis, v. 9, n. I, p. 92-100, jan. 2007.

GILL, D. et al. Competitive orientations and motives of adult sport and exercise participants. Journal of Sport Behavior, New York, v. 19, p. 307-318, dec. 1996.

HASKELL, W. L.; BLAIR, S. N.; HILL, J. O. Physical activity: Health outcomes and importance for public health policy. Preventive Medicine, New York, v. 49, n. 4, p. 280-282, oct. 2009.

HIRSCHBRUCH, M. D.; FISBERG, M.; MOCHIZUKI, L. Consumo de suplementos por jovens frequentadores de academias de ginástica em São Paulo. Revista Brasileira de Medicina do Esporte, Niterói, v. 14, n. 6, p. 539-43, nov./dez. 2008.

KILPATRICK, M.; HEBERT, E.; BARTHOLOMEW, J. College students's motivation for physical activity: differentiating men's and women's motives for sport participation and exercise. Journal of American College Health, Washington, v. 54, n. 2, p. 84-94, sep./oct. 2005.

KNUTH, A. G. et al. Conhecimento de adultos sobre o papel da atividade física na prevenção e tratamento de diabetes e hipertensão: estudo de base populacional no Sul do Brasil. Cadernos de Saúde Pública, Rio de Janeiro, v. 25, n. 3, p. 513-520, mar. 2009.

LEGNANI, R. F. S. Fatores motivacionais associados à prática de exercícios físicos em universitários. 139 f. Dissertação (Mestrado em Educação Física), Departamento de Educação Física, Universidade Estadual de Londrina, Londrina, 2009.

MALTBY, J.; DAY, L. The relationship between exercise motives and psychological well-being. The Journal of Psychology, Provincetown, v. 135, n. 6, p. 65I-660, nov. 2001.

MARKLAND, D.; HARDY, L. The Exercise Motivations Inventory: Preliminary development and validity of a measure of individuals' reasons for participation in regular physical exercise. Personality \& Individual Differences, Saarbruecken, v. I 5, n. 3, p. 289-296, sep. 1993.

MARKLAND, D.; INGLEDEW, D. K. The measurement of exercise motives: Factorial validity and invariance across gender of a revised Exercise Motivations Inventory. British Journal of Health Psychology, Leicester, v. 2, p. 361-376, nov. 1997. 
MARTÍNEZ-LÓPEZ, E. La salud como motivación para la práctica de actividad física en personas adultas. IATREIA, Medellín, v. 16, n. I, p. 32-43, mar. 2003.

MAZO, G. Z.; CARDOSO, F. L.; AGUIAR, D. L. Programa de hidroginástica para idosos: motivação, autoestima e autoimagem. Revista Brasileira Cineantropometria e Desempenho Humano, Florianópolis, v. 8, n. 2, p. 67-72, abr./jun. 2006.

MOUTÃO, J. M. R. P.; ALVES, J.; SILVA C. Motivos para a prática de exercício. In: SEABRA, A. et al. Investigação em Exercício e Saúde. Rio Maior: ESDRM-IP, 2003. p. 20 I-2I 0.

NETZ, Y.; RAVIV, S. Age differences in motivational orientation toward physical activity: an application of social cognitive theory. Journal of Psychology, Provincetown, v. I38, n. I, p. 35-48, jan. 2004.

REICHERT, F. F. Barreiras à prática de atividades físicas: prevalência e fatores associados. 120 f. Dissertação (Mestrado em Epidemiologia), Departamento de Medicina Social, Universidade Federal de Pelotas, Pelotas, 2004.

ROJAS, P. N. C. Aderência aos programas de exercícios físicos em academias de ginástica na cidade de Curitiba - PR. 203 f. Dissertação (Mestrado em Educação Física), Centro de Educação Física e Desporto, Universidade Federal de Santa Catarina, Florianópolis, 2003.

ROSE, E. A.; MARKALND, D.; PARFITT, G. The development and initial validation of the Exercise Causality Orientations Scale. Journal of Sports Sciences, London, v. 19, n. 6, p. 445462, jun. 2001.

RYAN, R. M.; DECI, E. L. Self-determination theory and the facilitation of intrinsic motivation, social development, and well-being. The American Psychologist, Washington, v. 55, n. I, p. 68-78, jan. 2000.

SALLIS, J. F. et al. Neighborhood environments and physical activity among adults in II countries. American Journal of Preventive Medicine, Washington, v. 36, n. 6, p. 484-490, jun. 2009.

SISSON, S. B.; KATZMARZYK, P. T. International prevalence of physical activity in youth and adults. Obesity Reviews, Oxford, v. 9, n. 6, p. 606-614, nov. 2008.

THOMPSON, J. L. Exercise in improving health vs. performance. Proceedings of the Nutrition Society, London, v. 68, n. I, p. 29-33, feb. 2009.

VELÁSQUEZ, M. T.; TORRES-NEIRA, D.; SÁNCHEZ-MARTíNEZ, H. Análisis psicológico de la actividad física en estudiantes de una universidad de Bogotá, Colombia. Revista de Salud Pública, Bogotá, v. 8, supl. 2, p. I-12, nov. 2006.

VUORI, I. Inactivity as a disease risk and health benefits of increased physical activity. In: OJA, P.; BORMS, J. Health Enhancing Physical Activity. 4. ed. Oxford: Meyer \& Meyer Sport. 2004. 
$X U, X$.; BIDDLE, S. Difference in motivation for adherence, gender and age. In: 2000 PRE-OLYMPIC CONGRESS SPORTS MEDICINE AND PHYSICAL EDUCATION INTERNATIONAL CONGRESS ON SPORT SCIENCE, 2000, Brisbane. Annals National Sports Information Centre, Brisbane: 2000. Disponivel em: < http://fulltext.ausport.gov.au/ fulltext/2000/preoly/abs436a.htm>. Acesso em: 30 outubro 2009.

Recebido: 25 jan. 2011 Aprovado: 05 jun. 2011

Endereço para correspondência:

Rosimeide Francisco Santos Legnani R. Salvador, 7|8 - Apto I0। - Bloco II

Bairro: Cajuru

Curitiba/PR

Cep: 82940- 160 